\title{
INTEGRAL HARNACK INEQUALITY
}

\author{
by MURALI RAO
}

(Received 18 November, 1983)

Introduction. Let $D$ be a domain in Euclidean space of $d$ dimensions and $K$ a compact subset of $D$. The well known Harnack inequality assures the existence of a positive constant $A$ depending only on $D$ and $K$ such that $(1 / A) u(x) \leq u(y) \leq A u(x)$ for all $x$ and $y$ in $K$ and all positive harmonic functions $u$ on $D$. In this we obtain a global integral version of this inequality under geometrical conditions on the domain. The result is the following: suppose $D$ is a Lipschitz domain satisfying the uniform exterior sphere condition-stated in Section 2. If $u$ is harmonic in $D$ with continuous boundary data $f$ then

$$
\int_{D}|u|(x) d x \leq C \int_{\partial D}|f| d s
$$

where $d s$ is the $d-1$ dimensional Hausdorff measure on the boundary $\partial D$. A large class of domains satisfy this condition. Examples are $C^{2}$-domains, convex domains, etc.

The lemma on which we base our proof states: For bounded domains satisfying the uniform exterior sphere condition solution of the Poisson equation with Dirichlet boundary conditions and constant forcing term has bounded gradient.

1. Generalities. Let $D$ be a bounded domain in Euclidean space of $d \geq 3$ dimensions. $G$ will denote its Green function: For all $x, y$

$$
G(x, y)=K(x, y)-H(x, y)
$$

where $K(x, y)=|x-y|^{-d+2}$ and $H(x, y)$ is the solution of the Dirichlet problem for $D$ with boundary data $K(\cdot, y)$. Write

$$
\sigma(x)=\int G(x, y) d y
$$

Then $\sigma$ satisfies the Poisson equation

$$
\begin{aligned}
\Delta \sigma & =-A_{d} \\
\sigma & =0 \text { at regular points of } \partial d .
\end{aligned}
$$

where $A_{d}=(d-2) 2 \pi^{d / 2} / \Gamma(d / 2)$.

For any positive Radon measure $m$ on $D$ the function $\int G(x, y) m(d y)$ is locally integrable in $D$ if it is finite at one point. Such a function is called a potential.

With the above notation and terminology we have

Proposrtion 1.1. Let $z$ be an arbitrary but fixed point in $D$. All potentials in $D$ are integrable on $D$ iff there is a constant $A$ depending only on $z$ and $D$ such that

$$
\sigma(y) \leq A G(z, y), \quad y \in D \text {. }
$$

Glasgow Math. J. 26 (1985) 115-120. 
Proof. Suppose all potentials in $D$ are integrable. If the assertion were false we could find a sequence $y_{n}$ such that $\sigma\left(y_{n}\right) \geq n^{2} G\left(z, y_{n}\right)$. If $m$ is the measure giving mass $\sigma\left(y_{n}\right)^{-1}$ to $y_{n}$ we have $\int G(z, y) m(d y)<\infty$. So $m$ determines a potential and this potential is integrable by assumption. However the integral of this potential is $\int \sigma(y) m(d y)=\infty$. A contradiction.

Conversely suppose (1.4) is valid. Let $p$ be the potential of the measure $m$. We have

$$
\int p(x) d x=\int \sigma(x) d m(x) \leq A p(z) \text {. }
$$

so that if $p(z)<\infty$ we are done. If $p(z)=\infty$ we proceed as follows: Take a ball contained in $D$ and containing $z$. The balayage $q$ of $p$ on the complement of $B$ is finite at $z$. $q$ is thus integrable. Since $p$ is locally integrable and equals $q$ off $B$ we find $p$ is integrable. The proof is complete.

COROLlary 1.2. If all potentials on a domain $D$ are integrable so are all positive harmonic functions.

Proof. Let $z$ be any point in $D$. From Proposition 1.1 there is a constant $A$ such that $\sigma(y) \leq A G(z, y)$. Let $u$ be positive harmonic. For any compact subdomain $E$, the reduit of $u$ on $E$ is a potential. The last inequality shows that the integral of this potential is bounded by $A u(z)$. As $E$ expands to $D$, these reduits increase to $u$. That completes the proof.

Proposition 1.3. Let $D$ be a bounded domain. For $x \in D$ let

Then

$$
d(x)=\operatorname{dist}(x, \partial D) .
$$

$$
|\operatorname{grad} \sigma(x)| \leq(d / d(x)) \sigma(x)
$$

where $d=$ dimension of space.

Proof. $\sigma$ satisfies the Poisson equation (see (1.3))

$$
\Delta \sigma=-C
$$

with Dirichlet boundary conditions. It follows that grad $\sigma$ is harmonic in $D$. Let $x \in D$ and $B$ the ball centre $x$ and radius $d(x)$. By the mean value property

$$
\begin{aligned}
\operatorname{grad} \sigma(x) & =\frac{1}{|B|} \int_{\boldsymbol{B}} \operatorname{grad} \sigma(y) d y \\
& =\frac{1}{|B|} \int_{\partial B} \sigma n d s
\end{aligned}
$$

where $|B|$ denotes volume of $B$; the last equality above being a consequence of the divergence theorem. Continuing

$$
|\operatorname{grad} \sigma(x)| \leq \frac{1}{|B|} \int_{\partial B} \sigma d s \leq \frac{d}{d(x)} \sigma(x)
$$

because $\sigma$ is superharmonic. The proof is complete. 
COROLLARY 1.4. Let $D$ be a bounded domain such that all points of $\partial D$ are regular. Then $\operatorname{grad} \sigma$ is bounded in $D$ iff

$$
\sigma(x) \leq \text { const } d(x)
$$

where $d(x)$ as in Proposition 1.3 denotes distance to the boundary.

Proof. Let grad $\sigma$ be bounded, $x \in D$ and $z \in \partial D$ satisfying $|x-z|=d(x)$. The line joining $x$ and $z$ is in $D ; z$ being regular $\sigma(z)=0$. We have

$$
\begin{aligned}
\sigma(x) & =\int_{0}^{1} \frac{d}{d t} \sigma(z+t(x-z)) d t \\
& =\int_{0}^{1}(x-z) \cdot \operatorname{grad} \sigma d t \\
& \leq|x-z|\|\operatorname{grad} \sigma\|_{\infty} .
\end{aligned}
$$

Proposition 1.5. Let $D$ be a bounded domain, $f$ measurable with $|f| \leq 1$. Then

$$
|\operatorname{grad} G f(x)| \leq \frac{d}{d(x)} G|f|(x)+\text { const }
$$

where const is independent of $f$. In particular if $\operatorname{grad} \sigma$ is bounded and all points of $\partial D$ regular then $\|\operatorname{grad} G f\|_{\infty} \leq M$ where $M$ is independent of $f$ and depends only on the dimension and volume of $D$.

Proof. Assume $f$ vanishes outside $D$ and put $\phi=K f . \phi$ is continuously differentiable [1] and

$$
G f=\phi-u
$$

where $u$ is the Dirichlet solution with boundary data $\phi$. Let us estimate the gradients of $\phi$ and $u$.

Writing $a=|x-y|, b=|z-y|$,

$$
\begin{aligned}
|K(x, y)-K(z, y)| & =\left|\frac{1}{a^{d-2}}-\frac{1}{b^{d-2}}\right| \\
& =|a-b| \sum_{i+j=d-1} 1 / a^{i} b^{i}
\end{aligned}
$$

For $i+j=d-1, a^{-i} b^{-j} \leq a^{-d+1}+b^{-d+1}$. We can continue from above

$$
|K(x, y)-K(z, y)| \leq|x-z| d\left[1 / a^{d-1}+1 / b^{d-1}\right] .
$$

$|f| \leq \mid$ and the integral

$$
\int_{D} \frac{1}{|\xi-y|^{d-1}} d y \leq \omega^{1-1 / d} \frac{d}{(d-1)^{1-1 / d}}|D|^{1 / d}
$$

where $|D|$ is the volume of $D, \omega$ the surface area of unit sphere and $d$ is the dimension. 
Integrating (1.9) and using (1.10)

$$
|\phi(\xi)-\phi(\eta)| \leq A|\xi-\eta|
$$

where $A$ depends only on the volume of $D$ and the dimension. We use (1.11) to estimate the gradient of $u$.

$u$ being harmonic in $D$, so is $\operatorname{grad} u$. Let $x \in D$ and $B$ the ball with centre $x$ and radius $d(x)$. By the mean value property

$$
\begin{aligned}
\operatorname{grad} u(x) & =\frac{1}{|B|} \int_{B} \operatorname{grad} u(y) d y \\
& =\frac{1}{|B|} \int_{\partial B} u \eta d s
\end{aligned}
$$

by the divergence theorem. Let $z \in \partial D$ such that $|x-z|=d(x)$. Continuing from above

$$
\begin{aligned}
|\operatorname{grad} u(x)| & =\left|\frac{1}{|B|} \int_{\partial B} u \eta d s\right| \\
& =\frac{1}{|B|} \int_{\partial B}(u(y)-\phi(z)) \eta d s \\
& \leq \frac{1}{|B|} \int_{\partial B}|u(y)-\phi(z)| d s
\end{aligned}
$$

Let $\tau(y)$ be a point on $\partial D$ satisfying

$$
|y-\tau(y)|=\operatorname{dist}(y, \partial D)
$$

$z$ being in $\partial D$,

$$
\begin{aligned}
& |y-\tau(y)| \leq|y-z| \leq 2 d(x) \\
& |\tau(y)-z| \leq|y-\tau(y)|+|y-z| \leq 4 d(x)
\end{aligned}
$$

Continuing from (1.12):

$$
\begin{aligned}
|\operatorname{grad} u(x)| \leq & \frac{1}{|B|} \int_{\partial B}|u(y)-\phi(\tau(y))| d s \\
& +\frac{1}{|B|} \int_{\partial B}|\phi(\tau(y))-\phi(z)| d s
\end{aligned}
$$

(1.8), (1.11) and (1.13) can be used to estimate the integrands above

$$
\begin{aligned}
|u(y)-\phi(\tau(y))| & \leq G|f|(y)+|\phi(y)-\phi(\tau(y))| \\
& \leq G|f|(y)+2 A d(x) \\
|\phi(\tau(y))-\phi(z)| & \leq 4 A d(x)
\end{aligned}
$$


Using these and continuing from (1.14) and remembering that $G|f|(y)$ is superharmonic

$$
|\operatorname{grad} u(x)| \leq \frac{d}{d(x)} G|f|(x)+6 D A
$$

Finally using (1.8), (1.11) and (1.15) we get (1.7). Since $G|f|(x) \leq \sigma(x)$, the second statement of the proposition follows from Corollary 1.4.

2. Domain condition. In this section we assume that the domain $D$ is nice enough to satisfy the uniform $R$-sphere condition:

There exists $R>0$ such that for each $z \in \partial D$ corresponds a point $\zeta$ such that $|\zeta-z|=R$ and the open ball with centre $\zeta$ and radius $R$ is completely contained in the complement of $D$.

This is a well known condition. See for example Courant-Hilbert [1]. Examples of such domains are domains with $\mathrm{c}^{2}$-boundaries convex domains etc.

Proposmion 2.1. Let $D$ be a domain satisfying the uniform $R$-sphere condition. Let $\sigma$ be as in (1.2). Then for $x \in D$

$$
|\operatorname{grad} \sigma(x)| \leq M
$$

where $M$ depends only the diameter of $D$, the dimension of space and $R$.

Proof. Let $x \in D$ and $z \in \partial D$ such that $|z-x|=d(x)$. By assumption there is a ball $B(\zeta, R)$ in the complement of $D$ and $|\zeta-z|=R$. The function

$$
\phi(y)=\frac{1}{R^{d-1}}-\frac{1}{|\zeta-y|^{d-1}}
$$

is positive and superharmonic in the complement of $B(\zeta, R)$. And for all $y \in D$

$$
\Delta \phi(y)=-(d-1)|\zeta-y|^{-d-1} \leq-(d-1)(A+R)^{-d-1}
$$

where $A=$ diameter of $D$. Since by (1.3) $\Delta \sigma=-A_{d}$ in $D, N \phi$ with $N=$ $A_{d}\left((A+R)^{d+1} / d-1\right)$ satisfies $\Delta(N \phi-\sigma) \leq 0$ in $D$. This means that $N \phi-\sigma$ is superharmonic in $D$ and since $\sigma=0$ on $\partial D, N \phi-\sigma \geq 0$ on $\partial D$. By the boundary minimum principle $N \phi \geq \sigma$ in $D$. Because $\phi(x) \leq R^{-d}|z-x|$ we obtain

$$
\sigma(x) \leq R^{-d} N|z-x| \mid
$$

Proposition (1.3) then gives (2.1).

THEOREM 2.2 (Harnack inequality). Let $D$ be a bounded Lipschitz domain satisfying the uniform exterior $R$-sphere condition. If $u$ is harmonic in $D$ with boundary data $f \geq 0$

$$
\int u d x \leq \frac{M}{A} d \int f d s
$$

where $d s$ is the $(d-1)$ dimensional Hausdorff measure on $\partial D, M$ and $A_{d}$ are given in (2.1) and (1.3). 
Proof. Let $A$ be a smooth subdomain of $D$ and $F \geq 0$ smooth on $R^{d}$. Then

$$
F+\frac{1}{A_{d}} \int_{D} G(x, y) \Delta F(y) d y=u
$$

where $u$ is the harmonic function in $D$ with boundary data $F$. Using Green's identity for $A$ and from (1.3)

$$
\int_{\mathrm{A}} \sigma \Delta F+A_{d} \int_{\mathrm{A}} F=\int_{\partial \mathrm{A}} \sigma \frac{\partial F}{\partial \eta}-\int_{\partial \mathrm{A}} F \frac{\partial \sigma}{\partial \eta}
$$

In this last equality if we let $A$ increase to $D$, and note that $\sigma=0$ on $\partial D$ :

$$
\int_{D} \sigma \Delta F+A_{d} \int_{D} F=-\lim \int_{\partial A} F \frac{\partial \sigma}{\partial \eta}
$$

Integrate both sides of (2.2) on $D$, compare with (2.3) and use (2.1) to get

$$
\int_{D} u \leq \frac{M}{A_{d}} \int_{\partial D} F d s
$$

where $d s$ is the Hausdorff dimensional measure on $\partial D$.

Remark. An easy conclusion from (2.4) is that for each $x \in D$ the harmonic measure at $x$ is absolutely continuous relative to $d s$ and has bounded density. Indeed let $m(x, d z)$ denote the harmonic measure at $x$ and put $m(d z)=\int_{D} m(x, d z) d x$. If $u$ and $F$ are as above

$$
\int_{\mathrm{D}} u=\int_{\partial \mathrm{D}} F d m
$$

and (2.4) immediately tells us that $m$ is absolutely continuous relative to $d s$ and has density bounded by $M / A_{d}$. On the other hand if $f \in L^{1}(m)$, then necessarily $f \in L^{1}(m(x, \cdot))$ for each $x \in D$ i.e. for each $x \in D, m(x, \cdot)$ has bounded density relative to $m$. In particular $m(x, \cdot)$ has bounded density relative to $d s$ as claimed.

\section{REFERENCE}

1. R. Courant and D. Hilbert Methods of Mathematical Physics. (Interscience). MATEMATISK INSTITUT AARHUS UNIVERSITY Aarhus, Denmark. 